\title{
MILP Model For Network Revenue Management In Airlines
}

\author{
Yoon Sook Song, Korean Air, Korea \\ Seong Tae Hong, Sangmyung University, Korea \\ Myung Sun Hwang, Korean Air, Korea \\ Moon Gil Yoon, Korea Aerospace University, Korea
}

\begin{abstract}
Seat inventory control is an important problem in revenue management which is to decide whether to accept or reject a booking request during the booking horizon in airlines. The problem can be modeled as dynamic stochastic programs, which are computationally intractable in network settings. Various researches have been tried to solve it effectively. Even though dynamic (and stochastic) programming (DP) models can be solved it optimally, they are computationally intractable even for small sized networks. Therefore, in practice, DP models are approximated by various mathematical programming models. In this paper, we propose an approximation model for solving airline seat inventory control problem in network environments. Using Linear Approximation technique, we will transform our problem into a concave piecewise LP model. Based on the optimal solution of ours, we suggest how to implement it for airline inventory control policies such as booking limits, bid-price controls and virtual nesting controls.
\end{abstract}

Keywords: Revenue Management, Airlines, Network RM, Seat Assignment, Booking Control

\section{INTRODUCTION}

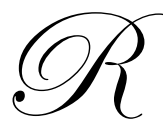

evenue Management has been recognized as an important problem in airlines for the last few decades. With limited resources of capacity and uncertain demands, airlines have tried to maximize their revenue from an efficient booking control mechanism, and developed various types of mathematical models to determine the best booking control strategies. Traditionally, the methodology can be divided into two groups: leg-based and network based RMs. Leg-based model is to optimize the passenger mix on a single leg flight. Whereas, network based model, network RM, is to determine an overall booking strategy for the entire network to maximize the revenue. A major flaw of leg-based models is that they only locally optimize booking control, whereas an airline should strive to maximize revenue from its network as a whole. There are significant revenue benefits from network RM. Talluri and Ryzin(2005) mentioned that network RM can increase the revenue significantly from $1.5 \%$ to $3 \%$ in airlines.

There are three well known booking control schemes for network RM: Partitioned Booking Limit(PBL), Virtual Nesting(VN) and Bid-Pricing(BP) controls. PBL which is an extension of a single leg control. It allocates a fixed amount of seats on each flight leg for every OD(Origin-Destination) demand. The allocated seats can be used for corresponding OD demand exclusively, that is, no other demand can use them. The second is a Virtual Nesting which is also an extension of a single leg control. In booking control, it uses single leg nesting controls for each flight leg. Thereby, it may take place the displacement problem. To adjust the displacement, we use virtual classes grouping together sets of ODFs(OD Fares) that use a given flight leg. Then we consider a indexing which provides a table mapping every ODF to a virtual class on each flight leg. After that, we assign ODF to a virtual class through indexing. The last one is Bid Price control in which the bid price can be interpreted as an estimate of the marginal cost to the network of consuming the next incremental unit of the flight capacity. In this control, we can not sell any ticket for a request unless its fare exceeds its bid-price. 
Network RM is how to manage the capacities of a set of connecting flights across a network., and has significant implementation and methodological challenges. On implementation, since there are lots of origindestination itinerary fare classes combinations, Network RM increases the complexity and volume of data that one must collect, store, and manage. Gathering the data for network RM is more difficult and complex than for a single leg RM. Network RM creates organizational challenges. Since revenue comes from an entire network, it is no longer easy to assign revenue responsibility for a single leg to a single user; revenue losses at one point in the network may be offset by gains elsewhere in the network. Creating organizational and incentive structure for supporting the objective of network RM is a challenging task. It is need to transit from a single leg-oriented RM organization to network-oriented one for improving the total revenue.

Due to a number of combinations of connecting flights and ODF, the problem for network RM is far more complex and difficult to solve than that for a single leg RM in the view of the problem scale, the data amount and the complexity of algorithms. Therefore, some modification techniques of the booking control for single-leg based RM have been considered for solving network RM problems.

Since determining an overall booking control strategy for the entire network is computationally intractable, researches have focused on developing efficient heuristics instead of optimal algorithms. There are many researches on network RM problems. Among them, Glover et al.(1982) proposed a mathematical programming model with deterministic demand. Curry(1990) extended Glover`s approach to a two-stage method aimed at incorporating the nesting of fare classes. Talluri and van Ryzin (1999) proposed a randomized linear programming (RLP) approach to incorporate the probabilistic nature of demand into the deterministic framework for network. More recently, Jiang(2006) considered Lagrangian relaxation method which provides both partitioned booking limits and Lagrangian multipliers for the bid-price policy.

In this paper, we focus on network revenue management problems with uncertain demands. We first explain our problem and existing seat assignment models for network RM in Section 2. Our problem has been considered as a complex stochastic programming problem due to the demand uncertainty. With some assumptions for demand distributions, we will show our problem can be formulated as a mixed Integer Programming model by applying a linear approximation technique. In Section 3, some computational experiments are performed to evaluate our model with randomly generated data set. Conclusion including further studies is given in Section 4 .

\section{SEAT ASSIGNMENT MODEL FOR MULTIPLE FLIGHT-LEGS}

A seat allocation problem with probabilistic demand in network environment can be described as follows. Given conditions are the entire network having multiple flight connections and the set of origin-destination itineraries with multiple origin-destination fare classes. The objective is to maximize the total revenue on the overall network. Decision variables are the number of seats allocating on each flight leg for each ODF.

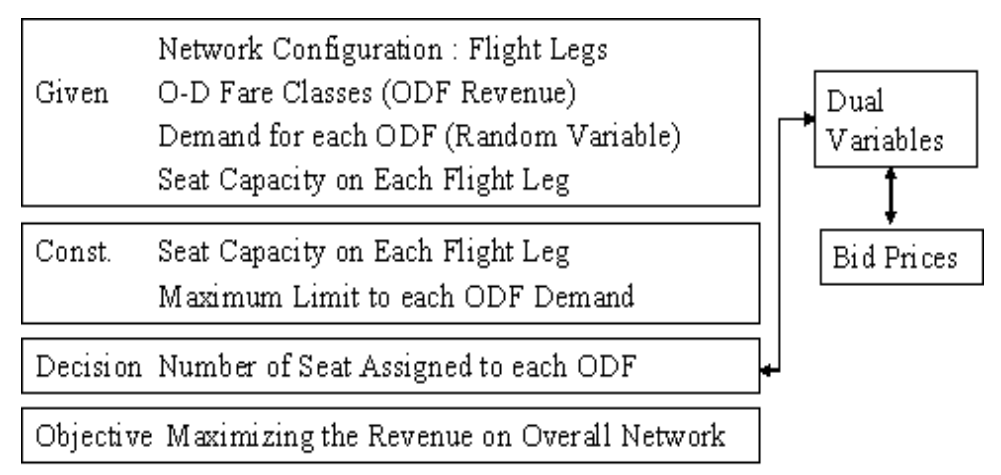

Figure 1. Seat allocation problems for Network RM 
Then, our problem can be modeled as a dynamic stochastic programming problem. However, due to the problem complexity, some approximations are considered to handle the problem easy such as deterministic or probabilistic programming models. When we consider the probabilistic demand for each ODF, the problem can be formulated as (PNLP) model.

To formulate an optimization model for our problem, we define the following variables:

$D_{j}:$ the demand for ODF $j$ (random variable, the maximum demand $\bar{D}_{j}$ ),

$r_{j}:$ the revenue per seat for ODF $j$,

$c_{i}$ : the capacity seat capacity of flight-leg $i\left(C=\left\{c_{i}\right\}\right)$,

$l_{j}, u_{j}: \min / \max$ number of seats to be assigned for ODF $j$ respectively,

$a_{i j}: 0,1$ index matrix which equal 1 if flight leg $i$ is used by ODF demand $j$ and 0 if not $\left(A=\left[a_{i j}\right]\right)$,

$x_{j}$ : the number of assigned seats for ODF $j$.

(PNLP) can be represented as follow:

$$
\begin{array}{rrr}
(P N L P) \quad \operatorname{Max} Z_{p}=\sum_{j} r_{j} E\left[\min \left(x_{j}, D_{j}\right)\right] & \\
\text { s.t. } & A x \leq C, \\
& l_{j} \leq x_{j} \leq u_{j}, & \forall j, \\
x_{j} & \geq 0, \quad \forall j .
\end{array}
$$

The objective is to maximize the expected revenue on overall network. The first constrain denotes the seat capacity on each flight leg, and the second represents the maximum limit of each ODF demand. Lots of algorithms have been proposed to solve (PNLP) in the view of a simplicity and a speed. Since (PNLP) is very complex to solve optimally, researchers have considered various types of approximation techniques based on network models and/or decomposition. When we replace the random demand variable $D_{j}$ by its expectation $E\left[D_{j}\right]$ for all $j$, then (PNLP) can be transformed to the deterministic LP(DLP) model which is computationally very efficient to solve. Even though (DLP) is far from (PNLP) and has a simple LP model, it provides some useful information such as partitioned limits and the dual variables for bid-prices, even it However, it does not reflect the demand uncertainty.

In this paper, we have proposed a linear approximation approach for solving the PNLP by exploiting its separable structure and its concavity. Applying approximation with assumptions for the random demand $D_{j}$, the expected value in the objective function in (PNLP) can be represented by deterministic terms. Then (PNLP) can be transformed into a Linear Programming model. The brief processes of Linear Approximation are given in Figure 2.

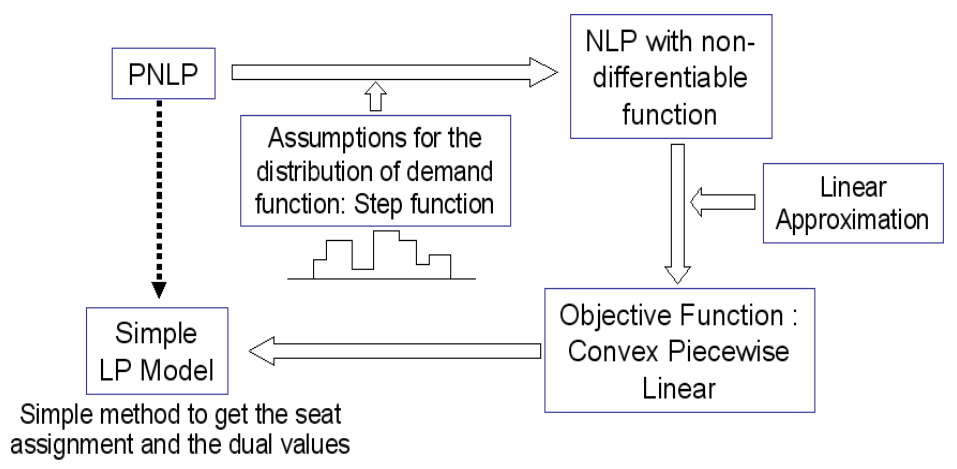

Figure 2. Linear Approximation Process 
In (PNLP), given the distribution of each demand, the expected revenue can be calculated by equation (1). However, since the objective has a non-linear function, the problem becomes very difficult to solve. Therefore, it is necessary to develop an efficient method for solving $\left[P_{0}\right]$ with ease. In this paper, we will apply a linear approximation techniques which was suggested by Szwarc(1964) for stochastic transportation. For linear approximation, we need some assumptions for the demand distributions of ODF demand:

- The demand is independent with each others and its distribution has a step function with the lower and the upper bounds.

- $\quad$ The interval between the lower $\left(L_{j}\right)$ and the upper bound $\left(\bar{D}_{j}\right)$ can be divided into $K$ sub-intervals.

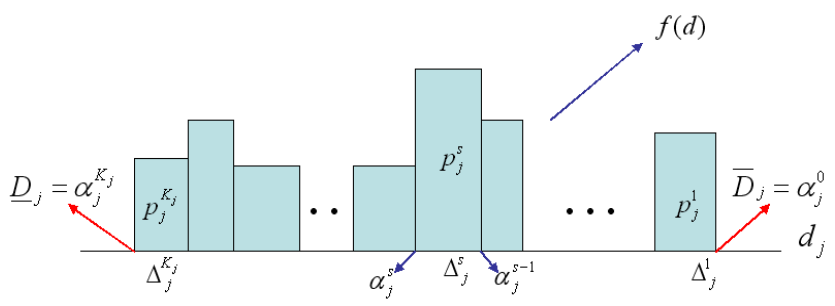

Figure 3. Demand Distribution for ODF $j$ : Step Function

Let $\Delta_{j}^{s} p_{j}^{s}$ be the interval size and the probability at $s$-th sub-interval for ODF demand $j$ respectively. Let $f(\theta)$ be the probability density function for ODF demand $j$, and define $\alpha_{j}^{s}$ as follow: $\alpha_{j}^{s}=\bar{D}_{j}-\sum_{k=1}^{s} \Delta_{j}^{k}$

Then we can see $\alpha_{j}^{0}=\bar{D}_{j}$ and $\alpha_{j}^{K}=L_{j}$. Since the demand distribution has a step function, the probability distribution function has a uniform distribution with p.d.f $f_{j}(\theta)=p_{j}^{s} / \Delta_{j}^{s}$ in the interval $\left[\alpha_{j}^{s}, \alpha_{j}^{s-1}\right]$.

Let consider $x_{j}$ has a value in the interval $\left[\alpha_{j}^{s}, \alpha_{i}^{s-1}\right]$, i.e., $\alpha_{j}^{s} \leq x_{j}<\alpha_{j}^{s-1}$. The expected value for the demand $j$ in the objective function (1) can be represented as follows:

$E\left[\min \left(x_{j}, D_{j}\right)\right]=\int_{\alpha_{j}^{K_{j}}}^{x_{j}} D f_{j}(D) d D+\int_{x_{j}}^{\alpha_{j}^{0}} x_{j} f_{j}(D) d D=z_{1 j}^{s}\left(x_{j}\right)+z_{2 j}^{s}\left(x_{j}\right)$

where, $z_{1 j}^{s}\left(x_{j}\right)=\int_{\alpha_{j}^{K_{j}}}^{x_{j}} D f_{j}(D) d D, z_{2 j}^{s}\left(x_{j}\right)=\int_{x_{j}}^{\alpha_{j}^{0}} x_{j} f_{j}(D) d D$

$z_{1 j}^{s}\left(x_{j}\right)=\int_{\alpha_{j}^{K_{j}}}^{x_{j}} D f_{j}(D) d D=\int_{\alpha_{j}^{s}}^{x_{j}} D \frac{p_{j}^{s}}{\Delta_{j}^{s}} d D+\int_{\alpha_{j}^{s+1}}^{\alpha_{j}^{s}} D \frac{p_{j}^{s+1}}{\Delta_{j}^{s+1}} d D+. .+\int_{\alpha_{j}^{K_{j}}}^{\alpha_{j}^{K_{j}-1}} D \frac{p_{j}^{K_{j}}}{\Delta_{j}^{K_{j}}} d D=\frac{p_{j}^{s}}{\Delta_{j}^{s}} \frac{\left(x_{j}^{2}-\alpha_{j}^{s 2}\right)}{2}+\sum_{i=s+1}^{K_{j}} \frac{\left(\alpha_{j}^{i}+\alpha_{j}^{i-1}\right)}{2} p_{j}^{i}$,

$z_{2 j}^{s}\left(x_{j}\right)=\int_{x_{j}^{K_{j}}}^{\bar{D}_{j}} D f_{j}(D) d D=\int_{x_{j}}^{\alpha_{j}^{s-1}} x_{j} \frac{p_{j}^{s}}{\Delta_{j}^{s}} d D+\int_{\alpha_{j}^{s-1}}^{\alpha_{j}^{s-2}} x_{j} \frac{p_{j}^{s-1}}{\Delta_{j}^{s-1}} d D+. .+\int_{\alpha_{j}^{1}}^{\alpha_{j}^{0}} x_{j} \frac{p_{j}^{1}}{\Delta_{j}^{1}} d D=x_{j}\left(\frac{p_{j}^{s}}{\Delta_{j}^{s}}\left(\alpha_{j}^{s-1}-x_{j}\right)+\sum_{i=1}^{s-1} p_{j}^{i}\right)$.

Since $z_{1 j}^{s}\left(x_{j}\right)$ and $z_{2 j}^{s}\left(x_{j}\right)$ are non-linear functions in the interval $\left[\alpha_{j}^{s}, \alpha_{i}^{s-1}\right]$, we replace them with linear functions $\bar{z}_{1 j}^{s}\left(x_{j}\right)$ and $\bar{z}_{2 j}^{s}\left(x_{j}\right)$ respectively by a linear approximation in the interval:

$$
\bar{z}_{1 j}^{s}\left(x_{j}\right)=z_{1 j}^{s}\left(\alpha_{j}^{s}\right)+\frac{\left(z_{1 j}^{s}\left(\alpha_{j}^{s-1}\right)-z_{1 j}^{s}\left(\alpha_{j}^{s}\right)\right)}{\Delta_{j}^{s}}\left(x_{j}-\alpha_{j}^{s}\right) \quad \bar{z}_{2 j}^{s}\left(x_{j}\right)=z_{2 j}^{s}\left(\alpha_{j}^{s}\right)+\frac{\left(z_{2 j}^{s}\left(\alpha_{j}^{s-1}\right)-z_{2 j}^{s}\left(\alpha_{j}^{s}\right)\right)}{\Delta_{j}^{s}}\left(x_{j}-\alpha_{j}^{s}\right)
$$


Then once $x_{j}$ has a value in the interval $\left[\alpha_{j}^{s}, \alpha_{i}^{s-1}\right]$, the expected value for the demand $j$ can be represented as a linear function approximately.

$$
r_{j} E\left[\min \left(x_{j}, D_{j}\right)\right] \approx H_{j}^{s}+h_{j}^{s}\left(x_{j}-\alpha_{j}^{s}\right) .
$$

$\left(H_{j}^{s}=r_{j}\left(z_{1 j}^{s}\left(\alpha_{j}^{s}\right)+z_{2 j}^{s}\left(\alpha_{j}^{s}\right)\right), \quad h_{j}^{s}=r_{j}\left(\frac{\left(z_{1 j}^{s}\left(\alpha_{j}^{s-1}\right)-z_{1 j}^{s}\left(\alpha_{j}^{s}\right)\right)}{\Delta_{j}^{s}}+\frac{\left(z_{2 j}^{s}\left(\alpha_{j}^{s-1}\right)-z_{2 j}^{s}\left(\alpha_{j}^{s}\right)\right)}{\Delta_{j}^{s}}\right)\right)$.

Let $x_{j}^{s}$ be a new variable defined in the interval $\left[\alpha_{j}^{s}, \alpha_{j}^{s-1}\right] x_{j}^{s}=\left(x_{j}-\alpha_{j}^{s}\right), 0 \leq x_{j}^{s} \leq \Delta_{j}^{s}$. Also, define $\pi_{j}^{s}\left(x_{j}^{s}\right)$ as follow; $\pi_{j}^{s}\left(x_{j}^{s}\right)=z_{1 j}^{-s}+z_{2 j}^{-s}=H_{j}^{s}+h_{j}^{s} x_{j}^{s}$. Since for demand $j, \pi_{j}^{s}\left(x_{j}^{s}\right)$ is a convex piecewise linear function for all interval, it is clear that if $x_{l}^{s}<\Delta_{i l}^{s}, x_{j}^{k}=0, k<s$, and if $x_{j}^{s}>0, x_{j}^{k}=\Delta_{j}^{k}, k>s$ in the optimal solution (Szwarc, 1964). Then the number of assigned seats for demand $j x_{j}$ can be obtained by $\sum_{s=1}^{K} x_{j}^{s}$. Therefore, we can represent approximately the expected revenue for demand $j$ over all the interval $\mathrm{s}=1,2, . ., \mathrm{K}$, as follows: $r_{j} E\left[\min \left(x_{j}, D_{j}\right)\right] \approx H_{j}^{K}+\sum_{s=1}^{K} h_{j}^{s} x_{j}^{s}$.

Since, without loss of generality, we can exclude the constant term $H_{j}^{K}$ in the optimization model, the objective function can be represented as a simple linear function. Consequently, applying a linear approximation technique, (PNLP) can be transformed to a linear programming model (P).

$$
\begin{array}{rll}
\text { (P) } \operatorname{Max} Z_{p}= & \sum_{j} \sum_{s} h_{j}^{s} x_{j}^{s} \\
\text { s.t. } & \sum_{j} \sum_{s} a_{i j} x_{j}^{s} \leq c_{i}, & \forall i, \\
x_{j}^{s} \leq \Delta_{j}^{s}, & \forall j, s, \\
x_{j}^{s} \geq 0, & \forall j, s .
\end{array}
$$

(P) is a simple LP with the upper bound constraints. We can solve it optimally by CPLEX or other mathematical programming tools with ease. Since (P) is a simple LP, it can provide the information for allocating seat to each flight leg as well as for calculating the bid price for each itinerary. Solving (P), we can get the optimal solution $\bar{x}_{j}^{s}$. The number of seat allocation for ODF demand $j$ is obtained by $x_{j}^{*}=\sum \bar{x}_{j}^{s}$. Since (P) gives a partitioned booking limit for each demand, it can not guarantee the revenue increase on the ${ }^{s}$ overall network. For the bid price policy, we need the information for dual values on flight legs.

\section{COMPUTATIONAL EXPERIMENTS}

To analyze the results of seat assignment for multiple flight demands, we will simulate lots of cases by using our models. In this paper, we compare our result with that of (DLP) for randomly generated demands on given multiple flight legs. Consider an example network with 6 airport, 5 flight legs and 11 itineraries (OD) as given in Figure 4. Airport 4 is a hub airport. We assume flights from airport 1,2, and 3 to the hub are short routes, while flights from the hub to the airport 5 and 6 are long routes. For each itinerary, we consider two types of fare classes and assume the demand of each fare class has the uniform distribution with lower and upper bounds. Table 1 gives itineraries, origins, destinations, fares and bounds for demand in our simulations. 


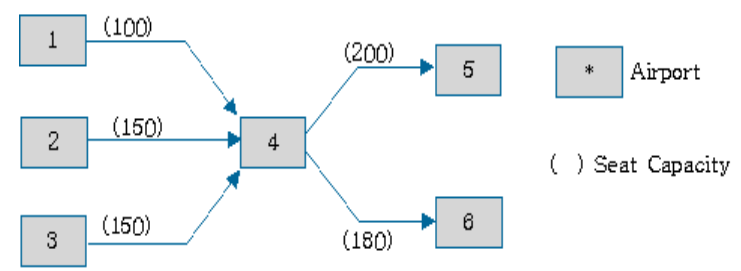

Figure 4 An example network

Table 1 Fares and demand by itinerary

\begin{tabular}{|c|c|c|c|c|c|}
\hline \multirow{2}{*}{ Itinerary } & \multirow{2}{*}{ Origin } & \multirow{2}{*}{ Destination } & \multirow{2}{*}{ Fares } & \multicolumn{2}{|c|}{ Demand } \\
\hline & & & & Lower Bound & Upper Bound \\
\hline \multirow{2}{*}{0} & \multirow{2}{*}{1} & \multirow{2}{*}{4} & 300 & 55 & 75 \\
\hline & & & 200 & 75 & 95 \\
\hline \multirow{2}{*}{1} & \multirow{2}{*}{1} & \multirow{2}{*}{5} & 500 & 15 & 35 \\
\hline & & & 300 & 25 & 45 \\
\hline \multirow{2}{*}{2} & \multirow{2}{*}{1} & \multirow{2}{*}{6} & 500 & 15 & 35 \\
\hline & & & 300 & 15 & 35 \\
\hline \multirow{2}{*}{3} & \multirow{2}{*}{2} & \multirow{2}{*}{4} & 200 & 55 & 75 \\
\hline & & & 150 & 115 & 135 \\
\hline \multirow{2}{*}{4} & \multirow{2}{*}{2} & \multirow{2}{*}{5} & 400 & 15 & 35 \\
\hline & & & 250 & 15 & 35 \\
\hline \multirow{2}{*}{5} & \multirow{2}{*}{2} & \multirow{2}{*}{6} & 400 & 15 & 35 \\
\hline & & & 200 & 25 & 45 \\
\hline \multirow{2}{*}{6} & \multirow{2}{*}{3} & \multirow{2}{*}{4} & 400 & 55 & 75 \\
\hline & & & 300 & 95 & 115 \\
\hline \multirow{2}{*}{7} & \multirow{2}{*}{3} & \multirow{2}{*}{5} & 500 & 15 & 35 \\
\hline & & & 350 & 15 & 35 \\
\hline \multirow{2}{*}{8} & \multirow{2}{*}{3} & \multirow{2}{*}{6} & 500 & 15 & 35 \\
\hline & & & 400 & 25 & 45 \\
\hline \multirow{2}{*}{9} & \multirow{2}{*}{4} & 5 & 800 & 55 & 75 \\
\hline & & 5 & 600 & 75 & 95 \\
\hline 10 & 4 & 6 & 800 & 75 & 95 \\
\hline 10 & 4 & 0 & 650 & 55 & 75 \\
\hline
\end{tabular}

With input parameters and the demand distributions, we can obtain the seat assignment for each ODF by applying our model. Using CPLEX6.0 on personal computer (Pentium D, 2.8GHz), we obtained the results given in Table 2. To get the booking limit for each ODF, we should consider a nesting structure. In our simulations, we adopt a hybrid nesting which combines a serial nesting and a parallel nesting.

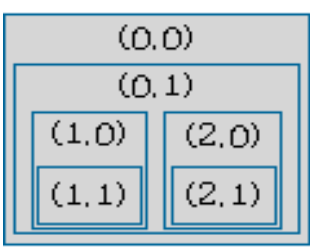

a) Flight Leg 1->4

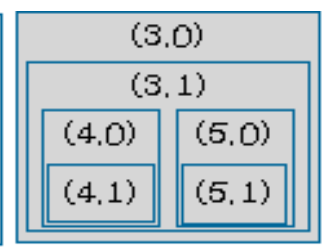

b) Flight Leg 2->4
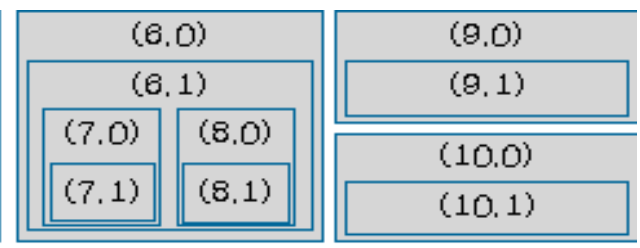

c) Flight Leg 3->4

d) Flight Leg 4->5(6)

Figure 5 Hybrid Nesting Structure - (Itinerary, Fare Class) 
With the booking limits for ODFs in Table 2, we simulate the seat control to maximize the total revenue. Table 3 gives the average simulation results for 500 trials including demand requests, accepted demands and total revenues. As we can find, the total revenue by using [P] is greater than that of [DLP]. As expected, the decrease of revenue in the short haul routes makes the increase of revenue in the long haul routes. Thereby the total revenue is improved. Comparing the results for [DLP], our model [P] recorded less sales volume in the number of seats (i.e. load factor), but ours makes more revenue in 500 simulation results. These results make sense in practices our model can be used for seat assignment in network RM effectively to maximize the total revenue.

Table 2 Booking Limit for each ODF

\begin{tabular}{|c|c|c|c|c|c|c|c|c|c|c|c|c|c|}
\hline \multirow{2}{*}{\multicolumn{3}{|c|}{$\begin{array}{c}\text { Flight-Leg } \\
\text { Itinerary }\end{array}$}} & \multicolumn{3}{|c|}{ [1->4] } & \multicolumn{3}{|c|}{$[2->4]$} & \multicolumn{3}{|c|}{ [3->4] } & \multirow{2}{*}{$\frac{[4->6]}{9}$} & \multirow{2}{*}{$\begin{array}{c}5->6] \\
10\end{array}$} \\
\hline & & & 0 & 1 & 2 & 3 & 4 & 5 & 6 & 7 & 8 & & \\
\hline \multirow{2}{*}[\mathbf{P}]{} & \multirow{2}{*}{ Fare Class } & 0 & 62 & 19 & 17 & 60 & 17 & 7 & 60 & 6 & 0 & 70 & 89 \\
\hline & & 1 & 2 & 0 & 0 & 60 & 0 & 0 & 84 & 0 & 0 & 88 & 67 \\
\hline \multirow{2}{*}{ DLP } & \multirow{2}{*}{ Fare Class } & 0 & 55 & 15 & 15 & 55 & 15 & 15 & 55 & 15 & 15 & 55 & 75 \\
\hline & & 1 & 0 & 15 & 0 & 55 & 10 & 0 & 60 & 0 & 5 & 75 & 55 \\
\hline
\end{tabular}

Table 3 Simulation Results

\begin{tabular}{|c|c|c|c|c|c|c|}
\hline \multirow{2}{*}{ Flight Leg } & \multirow{2}{*}{$\begin{array}{c}\text { Demand } \\
\text { Request }\end{array}$} & \multicolumn{2}{|c|}{ Sales-No. of Seats } & \multicolumn{3}{c|}{ Sales-Revenue } \\
\cline { 3 - 7 } & {$[\mathbf{D L P}]$} & {$[\mathbf{P}]$} & {$[\mathbf{D L P}]$} & $26,471.8$ & -3.04 \\
\hline$[1->4]$ & 238 & 90 & 88 & $27,302.6$ & $27,544.6$ & -4.79 \\
\hline$[2->4]$ & 275 & 135 & 134 & $28,931.4$ & $47,228.4$ & -7.03 \\
\hline$[3->4]$ & 258 & 135 & 135 & $50,799.4$ & $94,459.6$ & 3.12 \\
\hline$[4->5]$ & 284 & 175 & 162 & $91,598.0$ & $101,236.9$ & 6.3 \\
\hline$[4->6]$ & 293 & 160 & 147 & $95,225.6$ & $296,941.3$ & 1.05 \\
\hline \multicolumn{7}{|c|}{ Total Revenue }
\end{tabular}

* Difference $(\%)=(1-[\mathrm{P}] /[\mathrm{DLP}]) \times 100$

Figures in each column indicate the average value of 500 trials.

\section{CONCLUSIONS}

We have addressed on the seat assignment problem for network RM. With some assumptions for the demand distribution and applying Linear Approximation technique, we can formulate our problem as a mixed Integer Linear Programming model which is ease to solve by various mathematical programming tools. In a simple network structure including a single hub, our model provides the optimal solution by applying CPLEX or other LP tools. To test the validity and the practical applicability of our model, we executed simulations with some randomly generated data. The computational results show that our model can be applied for providing a good guideline to make an efficient seat control for multiple flight legs in airlines.

In this paper, we focused on network RM problems in airlines, and tried to formulate the problem as MILP model by applying the linear approximation technique with some assumptions effectively. To enhance the applicability of our model in practice, it needs to develop more comprehensive models including cancellations, noshows and behavioral factors in air travelers.

\section{AUTHOR INFORMATION}

Yoon-Sook Song is a deputy general manager of Revenue Management Team, Dept of Passenger route management, Korean air. She received a Ph.D. in Management Science from Korea Aerospace University; 'Integrated Seat Allocation Models for Airline Revenue Management' in 2008. Since 1998, she has been with Korean air; as an airport staff, flight controller and manager of route of south Asia and America. Her research interests in revenue management for airlines and service industries. 
Seong Tae Hong is a professor of Marketing at SangMyung University in Korea. He received his BS and MS in Business Administration from Seoul National University, and PhD in Marketing from the Graduate School of Seoul National University. Prior to joining current position, he had worked for Korea Telecom Research Institute as a senior researcher. His research interests include the introduction and strategy development of high-tech products or services. He has also conducted researches on demand forecasting and consumer behavior of newly-introduced IT and telecom services. He has published in some journals.

Myung-Sun, Hwang is a Vice Chief Officer \& Managing Vice President, Passenger Sales \& Marketing, Korean Air. He received a MBA, Graduate School of International Trade \& Logistics, In Ha University, Korea in 2009. Since 1983, he has been with Korean air; as a Team Manager of Passenger Sales \& Marketing, Japan HQ Office and Passenger Sales \& Marketing - Korea-Japan Route, HDQ Office, and Regional Manager \& Vice President of Pusan Sales Office and Regional HDQ Office of Korea. His research interests in the whole of Airline business.

Moon-Gil Yoon is a professor of Management Science in the Department of Business Administration, Korea Aerospace University in Korea. He received a Ph.D. in Management Science from Korea Advanced Institute of Science and Technology. Since 1992, he has been with Korea Aerospace University. He was a visiting scientist at the IBM Thomas J. Watson Research Center, a visiting professor at Ohio State University in US, at Osaka University in Japan, and at CSIRO in Australia. His current research interests are in the areas of e-Business models and revenue management for airlines and service industries.

\section{REFERENCES}

1. Belobaba, P., "Application of a probabilistic decision model to airline seat inventory control," Operations Research Vol. 37 (1989), pp183-197.

2. Bertsimas, D. and Popescu, I., "Revenue management in a dynamic network environment," Transportation Science Vol. 37(2003), pp257-277.

3. de Boer, S., Freling, R. and Piersma, N., "Mathematical programming for network revenue management revisited," European Journal of Operational Research Vol. 37(2002), pp72-92.

4. Jiang, H., "A Lagrangian relaxation approach for network inventory control of stochastic revenue management with perishable commodities," Journal of the Operational Research Society, Vol. , No. 1(2006), pp1-9.

5. $\quad$ McGill, J.I., van Ryzin, G.J., "Revenue management: research overview and prospects," Transportation Science Vol. 33 (1999), pp233-256.

6. $\quad$ Szwarc, W., "The transportation problem with stochastic demand," Management Science 11 (1964), pp3350.

7. Talluri, K. and van Ryzin, G., "An analysis of bid-price controls for network revenue management," Management Science Vol. 44(1999), pp1577-1593.

8. Talluri, K. and van Ryzin, G., "A randomized linear programming method for computing network bid prices," Transportation Science Vol. 33(1999), pp207--216.

9. Talluri, K. and van Ryzin, G., The Theory and Practice of Revenue Management. Springer Science+Business Media, Inc. New York, 2005.

10. Yoon, M.G., "Linear approximation model for network RM, " AGIFORS - Joint Revenue Management, Distribution and Cargo Study Group Conference, May 14-17, 2007, Jeju, Korea. 